\title{
Systems Biology Approaches for Host-Fungal Interactions: An Expanding Multi-Omics Frontier
}

\author{
Luka Culibrk, Carys A. Croft, and Scott J. Tebbutt ${ }^{1-3}$
}

\begin{abstract}
Opportunistic fungal infections are an increasing threat for global health, and for immunocompromised patients in particular. These infections are characterized by interaction between fungal pathogen and host cells. The exact mechanisms and the attendant variability in host and fungal pathogen interaction remain to be fully elucidated. The field of systems biology aims to characterize a biological system, and utilize this knowledge to predict the system's response to stimuli such as fungal exposures. A multi-omics approach, for example, combining data from genomics, proteomics, metabolomics, would allow a more comprehensive and pan-optic "two systems" biology of both the host and the fungal pathogen. In this review and literature analysis, we present highly specialized and nascent methods for analysis of multiple -omes of biological systems, in addition to emerging single-molecule visualization techniques that may assist in determining biological relevance of multi-omics data. We provide an overview of computational methods for modeling of gene regulatory networks, including some that have been applied towards the study of an interacting host and pathogen. In sum, comprehensive characterizations of hostfungal pathogen systems are now possible, and utilization of these cutting-edge multi-omics strategies may yield advances in better understanding of both host biology and fungal pathogens at a systems scale.
\end{abstract}

\section{Introduction}

I NVASIVE FUNGAL INFECTIONS (IFIs) are caused by opportunistic fungi such as the filamentous Aspergillus fumigatus or the yeasts Candida albicans and Cryptococcus neoformans (Enoch et al., 2006). Though not typically a concern in healthy individuals, IFIs are able to afflict ill or immunocompromised patients severely, including individuals with leukemia, transplant recipients, and those with HIV/AIDS (Comely et al., 2015; de Oliveira et al., 2014; Klingspor et al., 2015; Neofytos et al., 2013).

The incidence of IFIs is increasing, and a large proportion of these IFIs are nosocomial (Beck-Sagué and Jarvis, 1993; Lehrnbecher et al., 2010). This is believed to be due to an increase in the population of immunocompromised individuals ( Lehrnbecher et al., 2010; Warnock, 2007). IFIs tend to have high mortality rates (Comely et al., 2015; Lehrnbecher et al., 2010), and as a result the improvement of current prophylactic and curative treatments is of increasing interest. It is essential that we understand the fundamental and dynamic biological interactions between host and fungal cells in order to advance the care and treatment of patients with IFIs.
Pathogenesis requires an interaction between a pathogen and its host. There are numerous examples of host-fungal interactions in the context of organisms causing IFIs. Aspergillus fumigatus has been shown to adhere to extracellular matrix of the lung as well as the surface of human lung epithelial cells (Gil et al., 1996, Sheppard, 2011). Additionally, the internalization of $A$. fumigatus spores by epithelial cells in vitro has been observed numerous times (Gomez et al., 2010; Oosthuizen et al., 2011; Wasylnka and Moore, 2003).

Candida albicans has been observed to invade host cells by inducing endocytosis (Dalle et al., 2010) or through active invasion, a process by which hyphae breach epithelial cell membranes (Dalle et al., 2010, Wächtler et al., 2011). It has been demonstrated that $C$. neoformans infects its host through an actin-dependent internalization mechanism (Guerra et al., 2014). These initial interactions often lead to other interactions between the host and fungus on numerous levels. Host-fungal interaction networks are extremely complex, as there are many inherent differences between mammalian cells and fungal cells. A comprehensive analysis of these networks would entail the use of "-omics"-wide techniques in order to capture both the drastic

\footnotetext{
${ }^{1}$ Centre for Heart Lung Innovation, St. Paul's Hospital, and ${ }^{3}$ Department of Medicine, Division of Respiratory Medicine, University of British Columbia, Vancouver, British Columbia, Canada.

${ }^{2}$ Prevention of Organ Failure (PROOF) Centre of Excellence, Vancouver, British Columbia, Canada.

(C) Luka Culibrk et al., 2016 Published by Mary Ann Liebert, Inc. This Open Access article is distributed under the terms of the Creative Commons Attribution Noncommercial License (http://creativecommons.org/licenses/by-nc/4.0/) which permits any noncommercial use, distribution, and reproduction in any medium, provided the original author(s) and the source are credited.
} 
and the subtle dynamic biological perturbations within both host and pathogen.

The study of various biological "-omics" is generally segregated into several major fields of high-throughput biology, notably genomics, transcriptomics, proteomics, and metabolomics. An ideal -omic analysis of an organism involves collection of complete and unbiased datasets representative of the entire set of biomolecules of interest. Techniques that do not select specific, or candidate, targets are of particular value as they permit identification of novel biological networks without prior knowledge. The use of high-throughput techniques such as these has recently become far more commonplace as they can provide a more complete picture of the complexities of an organism's or cell's responses to experimental or environmental conditions. More prevalent quantitative techniques such as western blots and reverse transcription quantitative PCR are only able to analyze specific targets and are thus unable to detect unexpected changes.

Historically, high-throughput biology has been associated with a prohibitive monetary cost, rendering many of these techniques inaccessible to most researchers. Despite this, highthroughput biology has undeniable potential for the systematic analysis of a complex biological system, such as a host-pathogen interaction (Fig. 1). Researcher uptake has been aided by an increase in affordability of several high-throughput biology techniques in recent years. A notable example is the price of commercial genome sequencing, with the full sequencing of a human genome now as low as US $\$ 1000$ (Veritas Genomics, 2015).

There are previous reviews on topics related to the hostfungal interaction (Durmuş et al., 2015; Horn et al., 2012; Santamaría et al., 2011), and our review builds upon these by outlining and integrating both experimental and computational methods in high-throughput biology. We place particular emphasis on recent innovations in technology that promise to yield valuable insights into the relatively limited field of host-fungal interactions.

\section{The "Omics"}

\section{Genomics}

Early genomic studies of host-pathogen interactions involved molecular genetics and classical mutagenesis-screeningcloning experiments in order to link a gene to a phenotype (Fromtling et al., 1982; Kwon-Chung et al., 1982; Sandhu et al., 1976). These studies allowed the identification of essential genes and virulence factors (Chang and KwonChung, 1994; Kwon-Chung et al., 1982). However, as these scientists did not have access to the same types of omicsplatforms and data as we have today, their studies were necessarily more limited in their scope.

Today there are many fully sequenced genomes with at least some annotations, allowing for a "forward genetics" approach, looking for protein-coding genes based on predicted

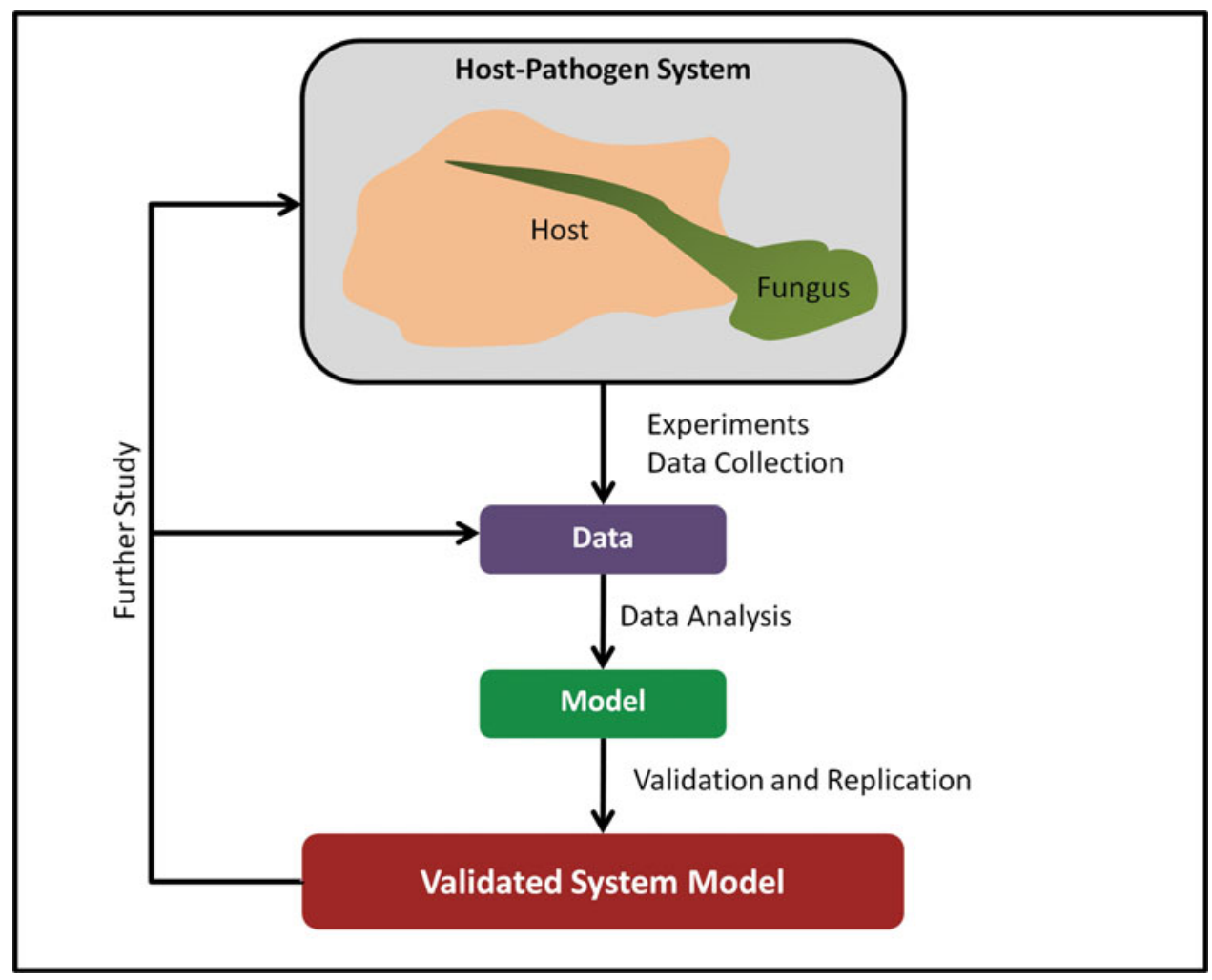

FIG. 1. Systems biology of dual-organism interactions. A defined experimental hostpathogen system is analyzed using high-throughput methods. Collected data are subjected to computational statistical analysis, and the results are analyzed using a number of bioinformatics technologies. Data analysis yields a model for the biological interaction. Replication results in a robust and validated model for the biological system. This validated model is then used to determine aspects of the system requiring further study and refinement. 
open reading frames, as opposed to simply a "reverse genetics" approach through the prediction of gene products based on inferred amino acid sequences from cDNAs (Paddison et al., 2002). The genomes of a number of species of pathogenic fungi have now been sequenced (Table 1) and forward genetics approaches can be applied to the study of host-fungal interactions.

Sequenced genomes have opened up many new avenues of research into the analysis of host-fungal interactions. Using more modern methods, functional analyses of the genomes of both organisms have been conducted alongside targeted genomic experiments to examine host-fungal interactions. These targeted experiments have also been able to make use of newly developed gene knockdown techniques. One such method is RNA interference (RNAi); the use of doublestranded RNA that binds to a specific mRNA transcript of interest, inhibiting translation (Hannon, 2002). RNAi has been used to great effect in the study of fungi alone (Eslami et al., 2014; Kalleda et al., 2013) and in the context of interaction with a host ( Chen et al., 2015b; Hu et al., 2008; Qin et al., 2011; Stroschein-Stevenson et al., 2009).

RNAi has numerous strengths when compared to a knockout by gene editing, such as simplicity and a relatively low cost, but comes with some significant challenges. Its primary weakness in some respects is its transience; high-turnover transcripts cannot be silenced effectively as the rapid production of mRNA quickly overwhelms the experimentally introduced silencing RNA (Larsson et al., 2010). Furthermore, RNAi is a knock-down, rather than a true knock-out. Protein function may not be completely abolished within the organism, and can affect experimental results (Moore et al., 2010).

A relatively new technique, the CRISPR/Cas9 gene editing system, has been developed for true gene knockout in eukaryotic cells (Wang et al., 2013). It has been shown that A. fumigatus and C. albicans are both compatible with the use of the CRISPR/Cas9 system (Fuller et al., 2015; Vyas et al., 2015), illustrating proof-of-concept for use of the CRISPR/ Cas9 system in the study of host-pathogen interactions. Despite its many potential applications, CRISPR/Cas9 is still in its infancy and has been plagued with issues such as low rates of uptake of the CRISPR/Cas9 machinery and off-target reactions. Further optimization of the basic molecular machinery of the CRISPR/Cas9 system is still underway (Zetsche et al., 2015).

Epigenetic modifications are another important aspect to consider when characterizing an organism's response, as they

Table 1. Sequenced Genomes of Pathogenic Fungi

Species Reference

Aspergillus sp.

Aspergillus fumigatus

Aspergillus nidulans

Aspergillus flavus

Candida sp.

Candida albicans

Cryptococcus sp.

Cryptococcus neoformans Loftus et al., 2005

Cryptococcus gatti

Nierman et al., 2005

Galagan et al., 2005

Nierman et al., 2015

Jones et al., 2004

D'Souza et al., 2011

Opportunistic pathogenic fungi are subdivided by genus, and individual species' genome sequences are cited as shown. have an effect on the rate of gene expression. A major technique used to analyze distribution of DNA-binding proteins is CHiP-seq; chromatin immunoprecipitation coupled to highthroughput parallel sequencing (Robertson et al., 2007). ChiP-seq has been used extensively to examine the epigenomic profiles of fungi such as $C$. neoformans and A. fumigatus, under various experimental conditions (Chung et al., 2014; Toh-e et al., 2015).

Other forms of epigenetic modification represent interesting fields of research; some, such as DNA methylation, have been investigated in pathogenic fungi (Liu et al., 2012; Mishra et al., 2011). Of note is the recently reported ability of some pathogenic bacteria to modify a host's gene expression through histone modifications and DNA methylation. A study by Sharma and colleagues (2015) determined that secreted bacterial methyltransferases were binding to host genes involved in the innate immune response. These observations corroborate a study that observed a downregulation of specific immune response genes due to a direct interaction between a pathogenic bacterial protein and host chromatin (Rennoll-Bankert et al., 2015).

In addition to its possible effects on pathogenesis, the ability to alter a host's epigenome could have arisen from host-pathogen co-evolution. Observation of this behavior in a bacterial host-pathogen system provides biological precedent for the possibility of a similar interaction between a fungus and its host. Such mechanisms could present targets for future study in antifungal treatment.

\section{Transcriptomics}

The transcriptome of a cell can provide useful insights into differences in an organism's gene expression under varying environmental conditions. The DNA microarray has allowed researchers to carry out analyses of the transcriptome of an organism for some time now (Schena et al., 1995), and has been applied extensively to the study of host-fungal interactions and changes in gene expression (Chow et al., 2007; Gomez et al., 2010; Kraus et al., 2004; Oosthuizen et al., 2011; Pukkila-Worley et al., 2005). Despite the many benefits, primarily ease of analysis and relatively low cost, microarrays have several limitations. Microarrays only detect selected gene transcripts that are based on the specific probes used on the array, preventing the discovery of novel transcripts and limiting the amount of data obtainable.

The more recently developed technique of RNA sequencing (RNA-seq) has provided a new way to analyze the transcriptome of an organism in more unbiased fashion (Mortazavi et al., 2008). Several studies have been conducted using RNA-seq to investigate dual-organism interactions (Chen et al., 2014; 2015a; Tierney et al., 2012), demonstrating its suitability as a tool for the analysis of host-fungal interactions from a transcriptomic perspective.

Validation of high-throughput transcriptomic data is essential for confidence in reported results. Quantitative PCR is a common method used to support the validity of RNAsequencing and microarray data (Chen et al., 2015b; Gomez et al., 2010, Oosthuizen et al., 2011). While the need for validation has been questioned by some (Fang and Cui, 2011), many journals still require some form of validation of results obtained using these high-throughput methods.

Whilst quantitative PCR is limited in its multiplexity, there are other recently developed techniques capable of quantifying 
up to a few hundred different targets, simultaneously. One notable example of this technology is the nanoString nCounter technology (Geiss et al., 2008), which can assay hundreds of different gene RNA targets within a single sample. Commercially available multiplexed quantitative PCR is similarly capable of assaying a large number of genes. These two technologies have been shown to be comparable to one another and to single-plex quantitative PCR (Prokopec et al., 2013). The ability to assay the expression of hundreds of genes of interest allows for a streamlined validation process.

Visualization of RNA molecules in situ is an area that has developed extensively in recent years. Fluorescent in situ hybridization (FISH) technology, wherein a probe complementary to a desired RNA target is bound to a fluorophore and allowed to hybridize with a cell sample prior to visualization with microscopy (Femino et al., 1998), has recently been refined into a number of specialized applications.

FISSEQ (fluorescent in situ sequencing) is a recently developed technique that enables in situ sequencing of RNA transcripts (Lee et al., 2014). This permits quantitative analysis of RNA transcripts within a single cell, and does not rely on a targeted search. As a new technique, FISSEQ still requires much optimization; the number of sequence reads is small compared to single-cell RNA-seq and requires a larger sample size and additional validation (Lee et al., 2015). Furthermore, the protocol appears to enrich for biologically active transcripts, introducing a potential for bias (Lee et al., 2015).

Another option would be for scientists to go "MERFISHing." Multiplexed error-robust FISH (MERFISH) is a novel technique recently developed by Chen and colleagues (2015a), which combines visualization of RNA molecules in situ similar to FISSEQ, with a combinatorial protocol, reducing the false discovery rate. MERFISH is a targeted search, limiting its ability to discover novel responses of the organism to an experimental condition. A potential solution would be the use of RNA-seq to identify targets of interest prior to conducting a MERFISH experiment.

To our knowledge, neither FISSEQ nor MERFISH has been applied towards the study of fungal pathogens; they do however show promise in the spatial resolution of transcriptomic perturbations, and could be coupled with the more standard RNA-seq or microarray methodologies to provide comprehensive insights.

\section{Proteomics}

While transcriptomic studies provide one view of gene expression, proteomics is another useful tool for the examination of a system's gene expression patterns. The gold standard for proteomic analysis, particularly in the field of systems biology, remains mass spectrometry (Sabidó et al., 2012). Large-scale quantitative proteomics is of interest as it enables the determination of relative expression changes between different conditions.

Three label-based, quantitative proteomics techniques are of particular note; SILAC, iTRAQ, and PUNCH-P. Stable isotope labeling of amino acids in cell culture (SILAC) compares protein expression between samples due to experimental conditions through the incorporation of stable, heavy isotopes of amino acids into a cell's proteins (Ong et al., 2002). Furthermore, SILAC can examine protein expression changes over specific time-points through "pulsing," a process whereby cells are transferred to SILAC media at certain stages of experimental treatment (Schwanhausser et al., 2009). SILAC is only compatible with cells that can be cultured for extended periods of time; pulsed-SILAC requires 6 hours at the minimum to provide meaningful data (Schwanhausser et al., 2009).

Isobaric tags for absolute and relative quantitation (iTRAQ) is another tag-based proteomics technique that tags peptide fragments at their N-terminus after digestion (Ross et al., 2004; Wiese et al., 2007). The specific advantage of iTRAQ is its versatility; unlike SILAC, iTRAQ is capable of analyzing protein expression changes over short periods of time, as incorporation of metabolic tags into proteins is not required. SILAC and iTRAQ have been used to study fungi in detail alone (Cagas et al., 2011; Georgianna et al., 2008; Zhang et al., 2015), and in a dual-organism setting (Reales-Calderón et al., 2013).

Puromycin-assisted nascent chain proteomics (PUNCH-P) is another recently developed technique able to detect newly synthesized proteins over short time periods with high accuracy through incorporation of puromycin into polypeptide chains (Aviner et al., 2013). Puromycin, a protein synthesis inhibitor, can be integrated into polypeptide chains when present in low concentrations. A chimeric puromycin-biotin "tag" then allows for purification of the tagged polypeptides by use of solid-phase streptavidin beads. Digestion of puromycin containing proteins prior to elution followed by mass spectrometry then yields analysis of newly synthesised peptides. While this technique has not yet been applied to mycology, PUNCH-P is a viable alternative to iTRAQ for analysis of short-term protein expression changes.

The localization of proteins is essential to understand their function and the interactions they have with proteins of the cell or pathogen. In addition, the localization of newly synthesized proteins can provide useful information about the proteomic response of one organism to another. Green fluorescent protein (GFP) constructs have been the method of choice for in situ visualization of proteins for some time (Chalfie et al., 1994). While GFP is useful for long-term study of steady-state systems, limited multiplexity and the inability to monitor differential expression decreases its usefulness for proteomic studies.

Multiplexed protein localization using GFP constructs has been investigated through a number of methods, such as subcellular fractionation for organelle-level spatial resolution (Zimmermann et al., 2010), or through the lengthy process of producing many GFP-protein constructs (Dénervaud et al., 2013). While indirect visualization through fractionation or direct visualization through transfection with engineered plasmid constructs is useful for determining spatial localisation of specific proteins upon a given treatment, the ability to directly visualize a variety of specific proteins and peptides in a high-throughput manner could be applied to numerous areas of research.

Surface sensing of translation (SUnSET) is a recently developed method that, like PUNCH-P, makes use of puromycin incorporation into a polypeptide chain, but follows this with anti-puromycin immunostaining for visualization of all newly synthesised proteins within a cell (Goodman et al., 2011; Schmidt et al., 2009). Recently, this has been refined to label specific proteins of interest in vivo by using an antibody pair: one anti-puromycin antibody, and one antibody against the target protein, and combining this approach with a proximity 
ligation assay (Tom Dieck et al., 2015). The use of puromycin for visualization is particularly advantageous as it allows for simultaneous PUNCH-P analysis of protein expression.

In addition to this antibody-based method, mass spectrometry can also be utilized for detailed analysis of a cell's proteome. A number of different methods have been developed for cellular mass spectrometry imaging, which have been described and summarized in a thorough review by Pól et al. (2010). The biologically relevant techniques described employ a precise laser that ionizes the surface of a biological sample, generating ions detectable through mass spectrometry.

Of particular interest is secondary ion mass spectrometry (SIMS), which provides a 2D resolution of below $1 \mu \mathrm{m}$, allowing for subcellular resolution (Pól et al., 2010). Stable isotope labeling has been combined with SIMS, resulting in multiisotope imaging mass spectrometry (MIMS) (Steinhauser et al., 2012). MIMS has been used to analyze protein content in single cells, including within dendritic spines (Brismar et al., 2014) and hair cell stereocilia (Zhang et al., 2012). The ability to analyze and map individual proteins of interest is a potentially powerful tool for elucidating host-pathogen interactions in dual-organism networks. If combined with visual transcriptomics techniques such as MERFISH, protein visualization methodologies could provide a valuable comparison between the distributions of transcripts and proteins in a cellular model.

Interactomics, a subset of proteomics, is particularly pertinent to host-pathogen interactions. The possibility that proteins of fungal and host cells not only interact but could be exchanged is intriguing and potentially quite valuable to our understanding of pathogenesis. The first challenge for dual-organism interactome analysis is to demonstrate the localization of host or pathogen proteins within the other organism. This can be accomplished using a SILAC-based technique, trans-SILAC (Rechavi et al., 2010). This method detects SILAC-tagged peptides within different cell samples, and has been applied towards the identification of human-human and bacterial-human protein delivery. This allows for the identification of inter-organism protein localization in a high-throughput, unbiased manner.

Direct observation of occurring protein-protein interactions is required subsequent to the identification of protein co-localization in an intercellular system. The yeast twohybrid system has been a standard for examination of protein-protein interactions (PPIs) for some time (Fields, 1993; Fields and Song, 1989). While this technique provides critical tools for interactome analysis, it has a number of caveats to consider. A high percentage of the PPIs predicted by the system are false positives, necessitating stringent validation (von Mering et al., 2002).

Integration of high-throughput proteomics into the analysis of PPIs whilst eliminating the precision challenges observed with the two-hybrid system has been achieved by protein correlation profiling-SILAC (PCP-SILAC) (Kristensen et al., 2012). PCP-SILAC essentially utilizes a guiltby-association approach by combining mass spectrometry with native chromatography; if two proteins migrate together in the chromatogram, they are assumed to be interacting (Fig. 2B). A combination of trans-SILAC and PCP-SILAC, a
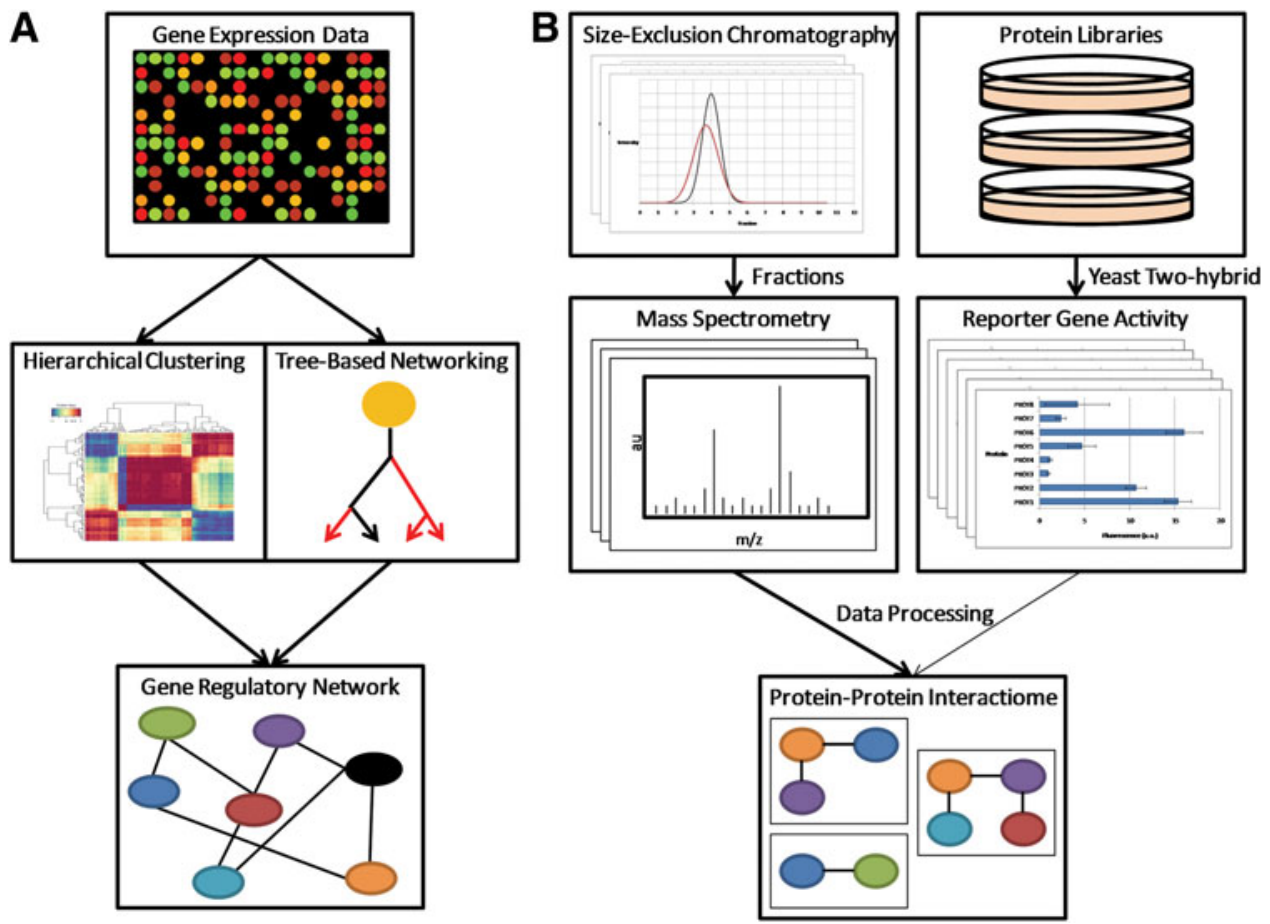

FIG. 2. Summary of modeling techniques. (A) Gene expression data are obtained from a microarray or RNA-seq. The data are processed using either hierarchical clustering or a tree-based machine-learning algorithm to identify network linkages. The final network is assembled based on the outputs of these algorithms. (B) PCP-SILAC (left) obtains interaction data from sizeexclusion chromatography and protein identity from mass spectrometry to determine proteinprotein interactions. Yeast two-hybrid obtains interaction data by constructing yeast that contain multiple transformants, and this interaction data is obtained in the form of reporter gene activity. 
"trans-PCP-SILAC" experiment would perhaps be capable of determining not only potential host-pathogen protein exchange, but also observation of inter-organism PPIs. Merging trans-SILAC and PCP-SILAC is feasible as they both use mass spectrometry to analyze samples. Such a study would yield protein expression data, possibly identify host-fungal protein exchange as well as determine protein-protein interactions between host-host, host-fungal, and fungal-fungal proteins.

\section{Metabolomics}

Metabolites are considered by some to be the final downstream effector molecules of the transcriptome and proteome. It therefore comes as no surprise that analysis of the metabolome would be valuable for the study of how fungi interact with their host, and vice-versa. Much like the genome, knowledge of an organism's metabolome provides a notable advantage to metabolic research. The human metabolome is freely available through the Human Metabolome Database (Wishart et al., 2007). No such database exists for any pathogenic fungi, an undeniable challenge in the analysis of complex fungal metabolomes.

High-throughput techniques exist that are capable of analyzing metabolomes, namely mass spectrometry and nuclear magnetic resonance (NMR). Mass spectrometry is capable of analyzing extremely low quantities of metabolites within a sample, allowing for detection of materials at low concentrations within the target cell. Unfortunately, mass spectrometry is not as precise in quantification of metabolites within a sample unless stable isotope labeling methods are used (Creek et al., 2012; Veenstra, 2012).

By contrast, NMR technology is capable of determining relative quantitation of metabolites with ease (Griffiths, 1998), although it struggles with a sensitivity several orders of magnitude lower than that of mass spectrometry (Veenstra, 2012; Webb, 2012). Both mass spectrometry (Choi et al., 2012, Han et al., 2012, 2013; Smedsgaard and Nielsen, 2005) and NMR (de Souza et al., 2013; Forseth et al., 2011, Sanchez and Wang, 2012) have been applied to fungal metabolomics.

Resolution of the localization of fungal metabolites has recently been made possible using MIMS (see above). Quantitative imaging of specific metabolites has been reported within yeast to a high degree of precision (Saiardi et al., 2014; Steinhauser and Lechene, 2013), demonstrating a proof-ofconcept for this method. To our knowledge, the metabolome has not been investigated in a dual-organism interaction setting. As the metabolome is capable of providing powerful insights into an organism's response, the integration of metabolomics into a host-fungal interaction study would be a highly valuable addition to other "-omics" data.

\section{Computational Modeling}

\section{Gene Network Inference: Dare to DREAM}

High-throughput datasets require a large degree of computational assistance for their analysis. These analyses may involve the use of transcriptomic, proteomic, or metabolomic datasets to generate interaction networks at the organism-organism level. These networks are of interest for the identification of key characteristics of host-pathogen interactions.

RNA-seq and microarray-based transcriptomic technologies allow for generation of transcriptome-wide gene ex- pression datasets. Gene network inference is an attempt to use high-throughput expression data to infer a regulatory network through a variety of statistical methods (Liu, 2015) (Fig. 2A). The DREAM (Dialogue on Reverse Engineering Assessment and Methods) challenges are a set of open challenges for researchers to utilize their own computational methods for the completion of a certain systems biology task.

One recent and notable example is the International Cancer Genome Consortium-The Cancer Genome Atlas DREAM Somatic Mutation Calling Challenge, in which participants were requested to simulate genomes of tumor cells, culminating in the development of an algorithm for prediction of cancer genomes (Ewing et al., 2015). DREAM challenge 5 , which compared the precision of 35 different methods for computing inferred gene regulatory networks, is the most recent competition examining the problem of network inference (Marbach et al., 2012).

The challenge provided gene expression data from microorganisms with known gene expression pathways; DREAM participants were instructed to attempt to reverse-engineer the gene regulatory network using their own software. The competitors of the challenge were subset into five categories based on methodology: regression, mutual information, correlation, Bayesian, and a group of assorted "others". Interestingly, the best performers were "GEne Network Inference with Ensemble of trees" (GENIE3), an algorithm using a tree-based methodology (Huynh-Thu et al., 2010), and an "ANalysis Of Variance" (ANOVA)-based method for nonlinear modeling (Küffner et al., 2012), two methods categorized as "others."

Other techniques have been developed outside the DREAM challenges, including the popular weighted gene correlation network analysis (WGCNA), which calculates a Pearson correlation coefficient between every gene in the dataset and weights each interaction based upon the strength of the correlation (Langfelder and Horvath, 2008). Further studies evaluated these network tools relative to one another and determined that GENIE3 provided a significant increase in precision when compared to all other evaluated tools (Madhamshettiwar et al., 2012). Other types of gene network modeling techniques have been used to survey potential gene regulatory networks in human pathogenic fungi (Altwasser et al., 2012, 2015).

It is worth noting that despite the progress in computational modeling, the top-scoring algorithms still returned a high rate of false positive interactions (Madhamshettiwar et al., 2012). GENIE3's random forest basis has been improved upon with the release of the iRafNet algorithm by allowing for the integration of data from outside sources, a capability that GENIE3 lacks (Petralia et al., 2015).

The DREAM challenges outline a key difficulty in the inference of genetic regulatory networks; computational modeling is inherently limited by the precision and replicability of high-throughput datasets. These datasets can be subset into two categories: analog, where the data is collected by observation of relative arbitrary units (such as fluorescent intensity), and digital data, which is a direct count of molecules within a sample. As these validations and comparisons were conducted utilizing microarray data, which is analog, they suffer both from the background noise and compressed linear scale. Indeed, most methods of high-throughput data collection, including the DNA microarray and spectrometry-based 
technologies are reliant on analog measurements, allowing only relative quantification of data at best, with significant noise introduced as a result.

Digital-based data, such as RNA-seq, provide a promising alternative to noisy analog datasets, despite still being only a relative quantification method. Models based upon this type of data show demonstrable improvement (Iancu et al., 2012). Despite this, current methods are still unable to provide a definitive model of gene regulatory networks (Ballouz et al., 2015). Nonetheless, it is difficult to obtain "perfect" datasets for these purposes that contain highly sensitive, precise data collected with a large dynamic range.

\section{Integration of the "-omics": Towards multi-omics biology}

Given the high rates of false network discovery, the development of methods to overcome these issues is essential. The integration of information and data outside of gene expression datasets lends a greater degree in confidence in an inferred network. Integration of prior network knowledge from the literature also provides increased accuracy to an inferred network (Ellwanger et al., 2014; Olsen et al., 2014). Based upon the central dogma of molecular biology, the proteome should provide a less biased view of gene expression compared to measurements of mRNA. Therefore integration of proteomics and transcriptomics into inference networks provides valuable correlation and correction of inferred models (Poultney et al., 2012).

Other types of valuable prior data exist, including proteinprotein interaction networks, CHiP-seq, and metabolomic data. Integration of some of these types of data has been conducted and demonstrated to increase confidence in an inferred network (Garcia-Reyero et al., 2014; Greenfield et al., 2013; Schöpke and Zeng, 2012). Although a few studies have examined the integration of metabolomic data into an inferred model of gene network inference, it remains a potentially powerful method of improving confidence in an inferred regulatory network.

Algorithms have been developed for multi-omic data integration, such as sparse generalized canonical correlation analysis (SGCCA) (Tenenhaus et al., 2014), which was specifically designed for this purpose. SGCCA has been applied to the integration of various types of datasets, including gene expression data from a microarray and DNA methylation (Wang et al., 2014), and microarray data with iTRAQ proteome data (Günther et al., 2014). The results from these studies are promising, indicating that SGCCA may be capable of providing excellent correlative assistance in the interpretation of systems-wide observations.

\section{Spatial Visualization}

Several of the techniques discussed earlier in this review require the use of microscopy. Confocal microscopy has been the staple for visualization of fluorescent molecules within cells for some time. Development of super-resolution fluorescent microscopy has permitted the imaging of single molecule fluorescence, and a number of specific methods have been developed (Betzig et al., 2006; Hess et al., 2006; Rust et al., 2006). This development has allowed for the implementation of quantitative fluorescent techniques such as FISSEQ and MERFISH, and provides the basis for their usage in a biological study.
The use of visualization techniques allows for the validation and observation of novel interactions that occur within an organism or between a pair of interacting organisms. Spatio-temporal modeling is the integration of time-course and spatial distribution. Currently, this has been applied to spatial resolution of the transcriptome between multiple regions of an organism, such as the different regions of the human brain (Kang et al., 2011) or different regions of a germ layer (Hashimshony et al., 2015).

Groundbreaking examples of protein-RNA co-localization research include studies into the role of Drosophila patterning genes, resulting in the discovery that these proteins and their transcripts are co-localized in the Drosophila embryo (Bergsten and Gavis, 1999; Berleth et al., 1988; Spirov et al., 2009). These studies were conducted using methods that can be effectively outperformed by newer techniques. FISSEQ and MERFISH are both capable of localizing RNA within a single cell to a high degree of accuracy (Chen et al., 2015a, Lee et al., 2015), and protein visualization techniques have likewise been drastically improved upon (Tom Dieck et al., 2015), allowing for the possibility of visualization of protein-mRNA co-localizations with increased resolution and multiplexity. These techniques allow both mRNA and protein localization to be correlated in-situ.

The mass spectrometry-based methods for visualisation (MIMS) of cellular components have been developed, and are capable of analysis of nucleic acids (Steinhauser et al., 2012), proteins (Brismar et al., 2014; Zhang et al., 2012), and metabolites (Saiardi et al., 2014). However, these methods are mass spectrometry-based, and as a result are quite expensive. Nevertheless, in a dual organism setting, such methods can potentially identify a myriad of novel interactions, such as protein-protein interactions between host and pathogen or changes in mRNA localization as a result of interaction with host or pathogen. The ability to visualize a dual organism interaction in this fashion could greatly elucidate our understanding of the interaction in a spatio-temporal context. The combination of previously discussed analytical methods with these visualisation techniques may provide key context for drawing conclusions for biological relevance (Fig. 3).

\section{Dual Organism Experiments}

The utilization of the methodologies described above, in a dual organism setting, has been limited. A notable example of one such experiment involved the inference of a trans-species interaction network between Mus musculus macrophages and $C$. albicans (Tierney et al., 2012). The experiment relied on dual organism RNA-seq to profile the transcriptome of C. albicans and M. musculus macrophages simultaneously. The authors then computationally inferred an interaction network demonstrating cross-species network regulation. To our knowledge, this is the only published example of a hostfungal experiment that used a modeling approach.

Dual organism transcriptomics has been utilized to characterize the transcriptomes of human lung airway cells and A. fumigatus simultaneously, however these studies could only draw conclusions based on gene ontology enrichment and direct changes in expression as they did not perform computational network modeling (Chen et al., 2015b; Gomez et al., 2010; Oosthuizen et al., 2011). The paucity of $A$. fumigatus functional gene annotations has been a limitation for 


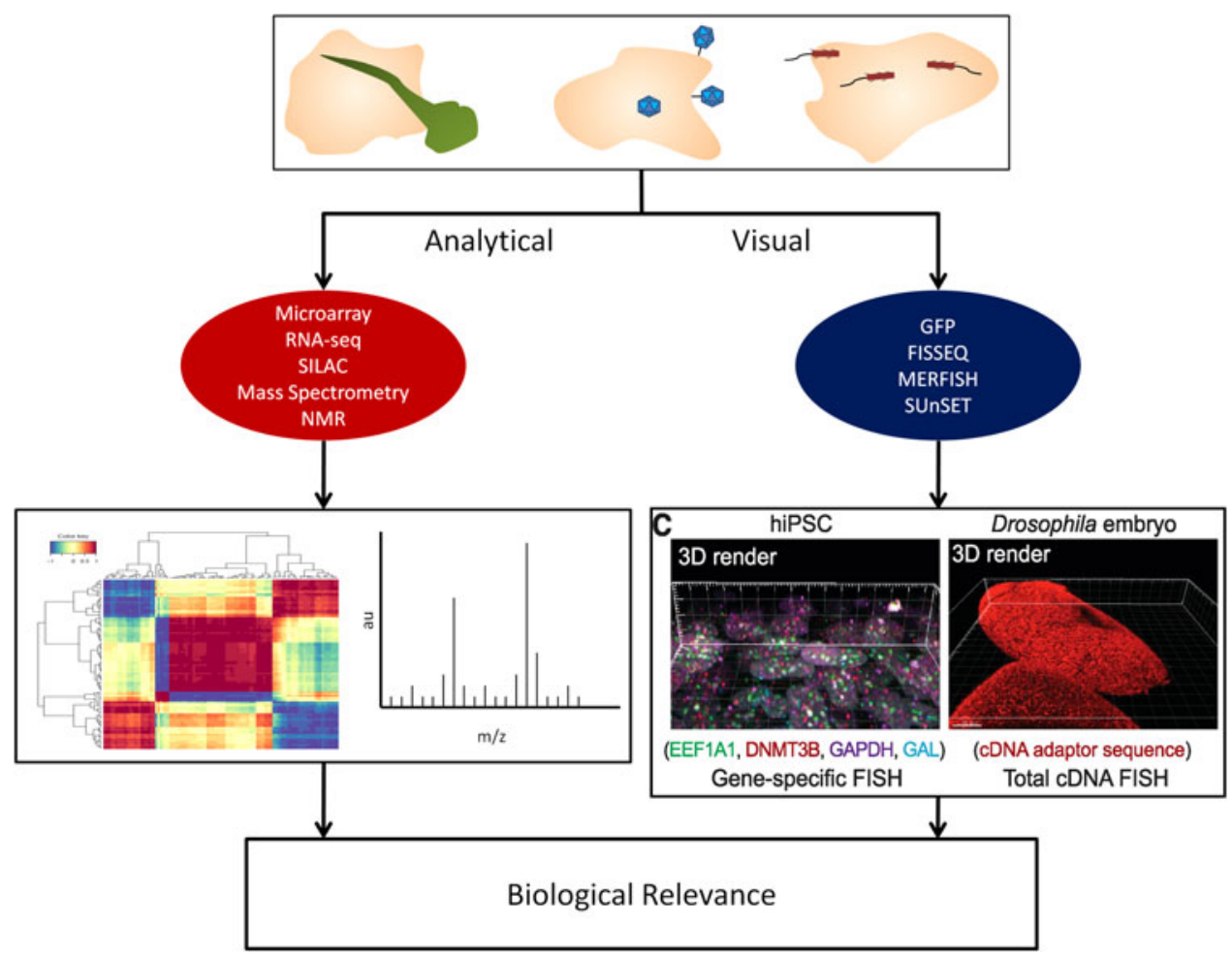

FIG. 3. Visualization methods offer novel options for observation of biological responses. A host-pathogen experiment can be analyzed using either analytical or visual methods. Analytical techniques generate large amounts of molecular data that require careful interpretation using statistical methods. Visual data produce visualizations of target molecules and systems. Box labeled "C'" adapted from Lee et al. (2012). Reprinted with permission from AAAS and authors.

these studies; many differentially expressed genes identified by Oosthuizen et al. (2011) lacked functional annotation. Nevertheless, such findings provide useful biological insight at a high level. Putative genes were identified with evidence for their expression, and in some cases differential expression, under specific experimental conditions. These observations allow for future studies to focus on these hitherto hypothetical genes to derive more functional insight. Hi-Jack, a tool designed for the analysis of simultaneous host-pathogen interactions using metabolomic data has been described (Kleftogiannis et al., 2015). This tool infers metabolic networks where a pathogen may be "hijacking" metabolites of its host, and has been used in a Mycobacterium tuberculosis model (Kleftogiannis et al., 2015). The application of such a framework to other "omics" areas could be highly informative. However, to our knowledge, Hi-Jack is the only tool developed specifically for use in a dual organism study.

\section{Challenges and the Road Ahead}

Significant challenges remain in the analysis of a dual organism host-fungal system. In terms of experimental design, fungal anatomy may lead to issues with the effectiveness of some experiments. The fungal cell wall represents an obstacle for many aspects of experimental protocols, such as the internalization of reagents, which may interfere with metabolic labeling necessary for techniques such as SILAC or SUnSET. Cell walls also interfere with lysis of fungal cells, which may be problematic when complete lysis is desired for maximum yield and low levels of technical bias.

While the DNA sequences, and thus amino acid sequences of genes and proteins vary enough to distinguish a fungal and mammalian molecule, many metabolites have no such restrictions. In this regard, network analysis via transcriptomic and proteomic analysis may be able to assist by identifying metabolic pathways of interest. While studies have managed to analyze the metabolome of various pathogenic fungi, it may prove to be exceedingly challenging to distinguish the fungal metabolome from a host metabolome, or vice-versa. Careful experimental design and precise technique is necessary to eliminate cross-contamination of processed samples, ensuring complete separation of host and fungal cells, or to verify the species that produced any given metabolite.

The introduction of a novel methodology into the laboratory also comes with significant challenges. Many techniques that we have discussed have only recently been reported. The potential power that these methods have is tempered by the fact that they have yet to be fully validated and assessed by the wider scientific community. Techniques allowing visualization of biomolecules, such as FISSEQ, have a large amount of inherent noise and significant false discovery rates, both of which present challenges that need to be solved for them to be used effectively.

These techniques also come with significant equipment and monetary requirements. Outfitting a microscope for FISSEQ analysis can cost up to US $\$ 20,000$ alone (Lee et al., 2014), in addition to other costs such as sequence library preparation. 
Many accepted and validated techniques in high-throughput biology, such as RNA-seq and mass spectrometry may still be prohibitively expensive for many research laboratories.

Despite the difficulties associated with them, modern tools have resulted in a number of new and exciting ways to analyse host-pathogen systems. Within the scope of any individual field of the "-omics," a number of questions have yet to be investigated in any sort of detail. The investigation into the inter-species regulatory network of $C$. albicans demonstrated the potential for the application of network inference between a host and fungus; this study is the only one of its kind within this field (Tierney et al., 2012).

Similar analyses directed towards other pathogenic fungi, such as A. fumigatus or Cryptococcus, or other types of host cells, would yield novel insights into the host-pathogen system. High-throughput proteomics is now capable of demonstrating protein-protein interactions between a host and pathogen, while simultaneously generating data on expression to corroborate transcriptomic observations by using PCPSILAC and trans-SILAC. Metabolomic integration would determine phenotypic consequences of fungal pathogenic infection and support biological pathway enrichment observed by proteomic and transcriptomic experiments.

Finally, direct observations of experimentally-hypothesised dual organism responses are now possible through multiple methods of fluorescent subcellular visualization. The "-omewide" nature of the techniques we have discussed provide valuable new ways to examine this field. These methods may be applied beyond model systems to clinical specimens so as to provide biomedical relevance that could assist in development of treatments for conditions such as invasive fungal infections.

Ultimately, clinical applications of -omics technologies at a patient level may require innovation beyond any research setting. For example, the Prosigna ${ }^{\circledR}$ prognostic assay, utilizing the nanoString nCounter ${ }^{\circledR}$ system has been FDA approved for use in a prognostic breast cancer perspective (US Food and Drug Administration, 2013). Instrument platforms such as the nCounter ${ }^{\circledR}$ system that allow simultaneous measurement across the "-omics" space are coming into production for research purposes and may drive forward ultimate clinical applicability. The continual development of "-omics" technology in a clinical setting will provide new avenues for clinical diagnostic and treatment options for invasive fungal infections.

\section{Acknowledgments}

We would like to thank the reviewers of this manuscript for their comments and input that have improved this review. We also thank Drs. Margo M. Moore and Tillie L. Hackett for their continued support. Funding for this project was provided in part by NSERC Discovery Grant RGPIN-2015-05043.

\section{Author Disclosure Statement}

The authors declare that no competing financial interests exist.

\section{References}

Altwasser R, Baldin C, Weber J, et al. (2015). Network modeling reveals cross talk of MAP kinases during adaptation to caspofungin stress in Aspergillus fumigatus. PLoS One 10, e0136932.
Altwasser R, Linde J, Buyko E, Hahn U, and Guthke R. (2012). Genome-wide scale-free network inference for Candida albicans. Front Microbiol 3, 51.

Aviner R, Geiger T, and Elroy-Stein O. (2013). Novel proteomic approach (PUNCH-P) reveals cell cycle-specific fluctuations in mRNA translation. Genes Dev 27, 1834-1844.

Ballouz S, Verleyen W, and Gillis J. (2015). Guidance for RNA-seq co-expression network construction and analysis: Safety in numbers. Bioinformatics 31, 2123-2130.

Beck-Sagué C, and Jarvis WR. (1993). Secular trends in the epidemiology of nosocomial fungal infections in the United States, 1980-1990. National Nosocomial Infections Surveillance System. J Infect Dis 167, 1247-1251.

Bergsten SE, and Gavis ER. (1999). Role for mRNA localization in translational activation but not spatial restriction of nanos RNA. Development 126, 659-669.

Berleth T, Burri M, Thoma G, et al. (1988). The role of localization of bicoid RNA in organizing the anterior pattern of the Drosophila embryo. EMBO J 7, 1749-1756.

Betzig E, Patterson GH, Sougrat R, et al. (2006). Imaging intracellular fluorescent proteins at nanometer resolution. Science 313, 1642-1645.

Brismar H, Aperia A, Westin L, et al. (2014). Study of protein and RNA in dendritic spines using multi-isotope imaging mass spectrometry. Surf Interface Anal 46, 158-160.

Cagas SE, Jain MR, Li H, and Perlin DS. (2011). Profiling the Aspergillus fumigatus proteome in response to caspofungin. Antimicrob Agents Chemother 55, 146-154.

Chalfie M, Tu Y, Euskirchen G, Ward WW, and Prasher DC. (1994). Green fluorescent protein as a marker for gene expression. Science 263, 802-805.

Chang YC, and Kwon-Chung KJ. (1994). Complementation of a capsule-deficient mutation of Cryptococcus neoformans restores its virulence. Mol Cell Biol 14, 4912-4919.

Chen KH, Boettiger AN, Moffitt JR, Wang S, and Zhuang X. (2015a). Spatially resolved, highly multiplexed RNA profiling in single cells. Science 1363, 1360-1363.

Chen Y, Toffaletti DL, Tenor JL, et al. (2014). The Cryptococcus neoformans transcriptome at the site of human meningitis. MBio 5, e01087-13.

Chen F, Zhang C, Jia X, et al. (2015b). Transcriptome profiles of human lung epithelial cells A549 interacting with Aspergillus fumigatus by RNA-Seq. PLoS One 10, e0135720.

Choi JN, Kim J, Kim J, Jung WH, and Lee CH. (2012). Influence of iron regulation on the metabolome of Cryptococcus neoformans. PLoS One 7, 1-10.

Chow ED, Liu OW, O'Brien S, aand Madhani HD. (2007). Exploration of whole-genome responses of the human AIDSassociated yeast pathogen Cryptococcus neoformans var grubii: nitric oxide stress and body temperature. Curr Genet 52, 137-148.

Chung D, Barker BM, Carey CC, et al. (2014). ChIP-seq and in vivo transcriptome analyses of the Aspergillus fumigatus SREBP SrbA reveals a new regulator of the fungal hypoxia response and virulence. PLoS Pathog 10, e1004487.

Comely O, Gachot B, Akan H, et al. (2015). Epidemiology and outcome of fungemia in a cancer cohort of the Infectious Diseases Group (IDG) of the European Organization for Research and Treatment of Cancer (EORTC 65031). Clin Infect Dis 61, 324-331.

Creek DJ, Chokkathukalam A, Jankevics A, Burgess KE V, Breitling R, and Barrett MP. (2012). Stable isotope-assisted metabolomics for network-wide metabolic pathway elucidation. Anal Chem 84, 8442-8447. 
Dalle F, Wächtler B, L'Ollivier C, et al. (2010). Cellular interactions of Candida albicans with human oral epithelial cells and enterocytes. Cell Microbiol 12, 248-271.

Dénervaud N, Becker J, Delgado-Gonzalo R, et al. (2013). A chemostat array enables the spatio-temporal analysis of the yeast proteome. Proc Natl Acad Sci USA 110, 15842-15847.

Durmuş S, Çakır T, Özgür A, and Guthke R. (2015). A review on computational systems biology of pathogen-host interactions. Front Microbiol 6, 235.

Ellwanger DC, Leonhardt JF, and Mewes H-W. (2014). Largescale modeling of condition-specific gene regulatory networks by information integration and inference. Nucleic Acids Res 42, 1-14.

Enoch DA, Ludlam HA, and Brown NM. (2006). Invasive fungal infections: A review of epidemiology and management options. J Med Microbiol 55, 809-818.

Eslami H, Khorramizadeh MR, Pourmand M, Moazeni M, and Rezaie S. (2014). Down-regulation of sidB gene by use of RNA interference in Aspergillus nidulans. Iran Biomed J 18, 55-59.

Ewing AD, Houlahan KE, Hu Y, et al. (2015). Combining tumor genome simulation with crowdsourcing to benchmark somatic single-nucleotide-variant detection. Nat Methods 12, 623-630.

Fang Z, and Cui X. (2011). Design and validation issues in RNA-seq experiments. Brief Bioinform 12, 280-287.

Femino AM, Fay FS, Fogarty K, and Singer RH. (1998). Visualization of single RNA transcripts in situ. Science 280, 585-590.

Fields S. (1993). The two-hybrid system to detect proteinprotein interactions. Methods 5, 116-124.

Fields S, and Song O. (1989). A novel genetic system to detect protein-protein interactions. Nature 340, 245-246.

Forseth RR, Fox EM, Chung D, Howlett BJ, Keller NP, and Schroeder FC. (2011). Identification of cryptic products of the gliotoxin gene cluster using NMR-based comparative metabolomics and a model for gliotoxin biosynthesis. J Am Chem Soc 133, 9678-9681.

Fromtling R, Shadomy HJ, and Jacobson ES. (1982). Decreased virulence in stable, acapsular mutants of Cryptococcus neoformans. Mycopathologia 79, 23-29.

Fuller K, Chen S, Loros J, and Dunlap J. (2015). Development of the CRISPR/Cas9 system for targeted gene disruption in Aspergillus fumigatus. Eukaryot Cell 14, 1073-1080.

Garcia-Reyero N, Ekman DR, Habib T, et al. (2014). Integrated approach to explore the mechanisms of aromatase inhibition and recovery in fathead minnows (Pimephales promelas). Gen Comp Endocrinol 203, 193-202.

Geiss GK, Bumgarner RE, Birditt B, et al. (2008). Direct multiplexed measurement of gene expression with colorcoded probe pairs. Nat Biotechnol 26, 317-325.

Georgianna DR, Hawkridge AM, Muddiman DC, and Payne GA. (2008). Temperature-dependent regulation of proteins in Aspergillus flavus: Whole organism stable isotope labeling by amino acids. J Proteome Res 7, 2973-2979.

Gil ML, Peñalver MC, Lopez-Ribot JL, O'Connor JE, and Martinez JP. (1996). Binding of extracellular matrix proteins to Aspergillus fumigatus conidia. Infect Immun 64, 5239-5247.

Gomez P, Hackett TL, Moore MM, Knight DA, and Tebbutt SJ. (2010). Functional genomics of human bronchial epithelial cells directly interacting with conidia of Aspergillus fumigatus. BMC Genomics 11, 358.

Goodman CA, Mabrey DM, Frey JW, Miu MH, Schmidt EK, Pierre P, and Hornberger TA. (2011). Novel insights into the regulation of skeletal muscle protein synthesis as revealed by a new nonradioactive in vivo technique. FASEB J 25, 10281039.
Greenfield A, Hafemeister C, and Bonneau R. (2013). Robust data-driven incorporation of prior knowledge into the inference of dynamic regulatory networks. Bioinformatics 29, 1060-1067.

Griffiths L. (1998). Assay by nuclear magnetic resonance spectroscopy: Quantification limits. Analyst 123, 1061-1068.

Guerra CR, Seabra SH, de Souza W, and Rozental S. (2014). Cryptococcus neoformans is internalized by receptor-mediated or "triggered" phagocytosis, dependent on actin recruitment. PLoS One 9, e89250.

Günther OP, Shin H, Ng RT, McMaster WR, McManus BM, Keown PA, Tebbutt SJ, and Lê Cao K-A. (2014). Novel multivariate methods for integration of genomics and proteomics data: Applications in a kidney transplant rejection study. OMICS 18, 682-695.

Han TL, Cannon RD, and Villas-Bôas SG. (2012). The metabolic response of Candida albicans to farnesol under hyphaeinducing conditions. FEMS Yeast Res 12, 879-889.

Han T-L, Tumanov S, Cannon RD, and Villas-Boas SG. (2013). Metabolic response of Candida albicans to phenylethyl alcohol under hyphae-inducing conditions. PLoS One 8, e71364.

Hannon GJ. (2002). RNA interference. Nature 418, 244-251.

Hashimshony T, Feder M, Levin M, Hall BK, and Yanai I. (2015). Spatiotemporal transcriptomics reveals the evolutionary history of the endoderm germ layer. Nature 519, 219-222.

Hess ST, Girirajan TPK, and Mason MD. (2006). Ultra-high resolution imaging by fluorescence photoactivation localization microscopy. Biophys J 91, 4258-4272.

Horn F, Heinekamp T, Kniemeyer O, Pollmächer J, Valiante V, and Brakhage AA. (2012). Systems biology of fungal infection. Front Microbiol 3, 1-20.

Hu G, Hacham M, Waterman SR, et al. (2008). PI3K signaling of autophagy is required for starvation tolerance and virulence of Cryptococcus neoformans. J Clin Invest 118, 1186-1197.

Huynh-Thu VA, Irrthum A, Wehenkel L, and Geurts P. (2010). Inferring regulatory networks from expression data using tree-based methods. PLoS One 5, 1-10.

Iancu OD, Kawane S, Bottomly D, Searles R, Hitzemann R, and McWeeney S. (2012). Utilizing RNA-Seq data for de novo coexpression network inference. Bioinformatics 28, 1592-1597.

Kalleda N, Naorem A, and Manchikatla RV. (2013). Targeting fungal genes by diced siRNAs: A rapid tool to decipher gene function in Aspergillus nidulans. PLoS One 8, e75443.

Kang HJ, Kawasawa YI, Cheng F, et al. (2011). Spatio-temporal transcriptome of the human brain. Nature 478, 483-489.

Kleftogiannis D, Wong L, Archer J, and Kalnis P. (2015). Hi-Jack: A novel computational framework for pathway-based inference of host-pathogen interactions. Bioinformatics 31, 2332-2339.

Klingspor L, Saeedi B, Ljungman P, and Szakos A. (2015). Epidemiology and outcomes of patients with invasive mould infections: A retrospective observational study from a single centre (2005-2009). Mycoses 58, 470-477.

Kraus PR, Boily M-J, Giles SS, et al. (2004). Identification of Cryptococcus neoformans temperature-regulated genes with a genomic-DNA microarray. Eukaryot Cell 3, 1249-1260.

Kristensen AR, Gsponer J, and Foster LJ. (2012). A highthroughput approach for measuring temporal changes in the interactome. Nat Methods 9, 907-909.

Küffner R, Petri T, Tavakkolkhah P, Windhager L, and Zimmer R. (2012). Inferring gene regulatory networks by ANOVA. Bioinformatics 28, 1376-1382.

Kwon-Chung KJ, Polacheck I, and Popkin TJ. (1982). Melaninlacking mutants of Cryptococcus neoformans and their virulence for mice. J. Bacteriol 150, 1414-1421. 
Langfelder P, and Horvath S. (2008). WGCNA: An R package for weighted correlation network analysis. BMC Bioinformatics 9, 559.

Larsson E, Sander C, and Marks D. (2010). mRNA turnover rate limits siRNA and microRNA efficacy. Mol Syst Biol 6, 433.

Lee JH, Daugharthy ER, Scheiman J, et al. (2015). Fluorescent in situ sequencing (FISSEQ) of RNA for gene expression profiling in intact cells and tissues. Nat Protoc 10, 442-458.

Lee JH, Daugharthy ER, Scheiman J, et al. (2014). Highly multiplexed subcellular RNA sequencing in situ. Science 343, 1360-1363.

Lehrnbecher T, Frank C, Engels K, Kriener S, Groll H, and Schwabe D. (2010). Trends in the postmortem epidemiology of invasive fungal infections at a university hospital. J Infect 61, 259-265.

Liu S-Y, Lin J-Q, Wu H-L, et al. (2012). Bisulfite sequencing reveals that Aspergillus flavus holds a hollow in DNA methylation. PLoS One 7, e30349.

Liu Z-P. (2015). Reverse engineering of genome-wide gene regulatory networks from gene expression data. Curr Genomics 16, 3-22.

Madhamshettiwar PB, Maetschke SR, Davis MJ, Reverter A, and Ragan M. (2012). Gene regulatory network inference: Evaluation and application to ovarian cancer allows the prioritization of drug targets. Genome Med 4, 41.

Marbach D, Costello JC, Küffner R, et al. (2012). Wisdom of crowds for robust gene network inference. Nat Methods 9, 796-804.

Von Mering C, Krause R, Snel B, Cornell M, Oliver SG, Fields S, and Bork P. (2002). Comparative assessment of large-scale data sets of protein-protein interactions. Nature 417, 399-403.

Mishra PK, Baum M, and Carbon J. (2011). DNA methylation regulates phenotype-dependent transcriptional activity in Candida albicans. Proc Natl Acad Sci USA 108, 11965-11970.

Moore CB, Guthrie EH, Huang MT-H, and Taxman DJ. (2010). Short hairpin RNA (shRNA): Design, delivery, and assessment of gene knockdown. Methods Mol Biol 629, 141-158.

Mortazavi A, Williams B, McCue K, Schaeffer L, and Wold B. (2008). Mapping and quantifying mammalian transcriptomes by RNA-Seq. Nat Methods 5, 621-628.

Neofytos D, Treadway S, Ostrander D, et al. (2013). Epidemiology, outcomes, and mortality predictors of invasive mold infections among transplant recipients: A 10-year, singlecenter experience. Transpl Infect Dis 15, 233-242.

De Oliveira RB, Atobe JH, Souza SA, and de Castro Lima Santos DW. (2014). Epidemiology of invasive fungal infections in patients with acquired immunodeficiency syndrome at a reference hospital for infectious diseases in Brazil. Mycopathologia 178, 71-78.

Olsen C, Fleming K, Prendergast N, et al. (2014). Inference and validation of predictive gene networks from biomedical literature and gene expression data. Genomics 103, 329-336.

Ong S-E, Blagoev B, Kratchmarova I, Kristensen DB, Steen H, Pandey A, and Mann M. (2002). Stable isotope labeling by amino acids in cell culture, SILAC, as a simple and accurate approach to expression proteomics. Mol Cell Proteomics 1, 376-386.

Oosthuizen JL, Gomez P, Ruan J, Hackett TL, Moore MM, Knight DA, and Tebbutt SJ. (2011). Dual organism transcriptomics of airway epithelial cells interacting with conidia of Aspergillus fumigatus. PLoS One 6, e20527.

Paddison PJ, Caudy AA, and Hannon GJ. (2002). Stable suppression of gene expression by RNAi in mammalian cells. Proc Natl Acad Sci USA 99, 1443-1448.

Petralia F, Wang P, Yang J, and Tu Z. (2015). Integrative random forest for gene regulatory network inference. Bioinformatics 31, 197-205.
Pól J, Strohalm M, Havlíček V, and Volný M. (2010). Molecular mass spectrometry imaging in biomedical and life science research. Histochem Cell Biol 134, 423-443.

Poultney CS, Greenfield A, and Bonneau R. (2012). Integrated inference and analysis of regulatory networks from multilevel measurements. Methods Cell Biol 110, 19-56.

Prokopec SD, Watson JD, Waggott DM, et al. (2013). Systematic evaluation of medium-throughput mRNA abundance platforms. RNA 19, 51-62.

Pukkila-Worley R, Gerrald QD, Kraus PR, et al. (2005). Transcriptional network of multiple capsule and melanin genes governed by the Cryptococcus neoformans cyclic AMP cascade. Eukaryot Cell 4, 190-201.

Qin Q-M, Luo J, Lin X, Pei J, Li L, Ficht TA, and de Figueiredo P. (2011). Functional analysis of host factors that mediate the intracellular lifestyle of Cryptococcus neoformans. PLoS Pathog 7, e1002078.

Reales-Calderón JA, Sylvester M, Strijbis K, Jensen ON, Nombela C, Molero G, and Gil C. (2013). Candida albicans induces pro-inflammatory and anti-apoptotic signals in macrophages as revealed by quantitative proteomics and phosphoproteomics. J Proteomics 91, 106-135.

Rechavi O, Kalman M, Fang Y, et al. (2010). Trans-SILAC: Sorting out the non-cell-autonomous proteome. Nat Methods 7, 923-927.

Rennoll-Bankert KE, Garcia-Garcia JC, Sinclair SH, and Dumler JS. (2015). Chromatin bound bacterial effector AnkA recruits HDAC1 and modifies host gene expression. Cell Microbiol. 17, 1640-1652.

Robertson G, Hirst M, Bainbridge M, et al. (2007). Genomewide profiles of STAT1 DNA association using chromatin immunoprecipitation and massively parallel sequencing. Nat Methods 4, 651-657.

Ross PL, Huang YN, Marchese JN, et al. (2004). Multiplexed protein quantitation in Saccharomyces cerevisiae using aminereactive isobaric tagging reagents. Mol Cell Proteomics 3, $1154-1169$.

Rust MJ, Bates M, and Zhuang X. (2006). Sub-diffraction-limit imaging by stochastic optical reconstruction microscopy (STORM). Nat Methods 3, 793-795.

Sabidó E, Selevsek N, and Aebersold R. (2012). Mass spectrometry-based proteomics for systems biology. Curr Opin Biotechnol 23, 591-597.

Saiardi A, Guillermier C, Loss O, Poczatek JC, and Lechene C. (2014). Quantitative imaging of inositol distribution in yeast using multi-isotope imaging mass spectrometry (MIMS). Surf Interface Anal 46, 169--172.

Sanchez JF, and Wang CCC. (2012). The chemical identification and analysis of Aspergillus nidulans secondary metabolites. Methods Mol Biol 944, 97-109.

Sandhu DK, Sandhu RS, Khan ZU, and Damodaran VN. (1976). Conditional virulence of a p-aminobenzoic acid-requiring mutant of Aspergillus fumigatus. Infect Immun 13, 527-532.

Santamaría R, Rizzetto L, Bromley M, et al. (2011). Systems biology of infectious diseases: A focus on fungal infections. Immunobiology 216, 1212-1227.

Schena M, Shalon D, Davis RW, and Brown PO. (1995). Quantitative monitoring of gene expression patterns with a complementary DNA microarray. Science 270, 467-470.

Schmidt EK, Clavarino G, Ceppi M, and Pierre P. (2009). SUnSET, a nonradioactive method to monitor protein synthesis. Nat Methods 6, 275-277.

Schöpke B, and Zeng A-P. (2012). Inference of responsive metabolic pathways from time-series transcriptomic data with 
consideration of the metabolic network structure. Integr Biol 4, 888-889.

Schwanhausser B, Gossen M, Dittmar G, et al. (2009). Global analysis of cellular protein translation by pulsed SILAC. Proteomics 9, 205-209.

Sharma G, Upadhyay S, Srilalitha M, Nandicoori VK, and Khosla S. (2015). The interaction of mycobacterial protein Rv2966c with host chromatin is mediated through non-CpG methylation and histone $\mathrm{H} 3 / \mathrm{H} 4$ binding. Nucleic Acids Res 43, 3922-3937.

Sheppard DC. (2011). Molecular mechanism of Aspergillus fumigatus adherence to host constituents. Curr Opin Microbiol 14, 375-379.

Smedsgaard J, and Nielsen J. (2005). Metabolite profiling of fungi and yeast: From phenotype to metabolome by MS and informatics. J Exp Bot 56, 273-286.

De Souza WR, Morais ER, Krohn NG, et al. (2013). Identification of metabolic pathways influenced by the G-protein coupled in Aspergillus nidulans. PLoS One 8, e62088.

Spirov A, Fahmy K, Schneider M, Frei E, Noll M, and Baumgartner S. (2009). Formation of the bicoid morphogen gradient: An mRNA gradient dictates the protein gradient. Development 136, 605-614.

Steinhauser ML, Bailey AP, Senyo SE, Guillermier C, Perlstein TS, Gould AP, Lee RT, and Lechene CP. (2012). Multiisotope imaging mass spectrometry quantifies stem cell division and metabolism. Nature 481, 516-519.

Steinhauser ML, and Lechene CP. (2013). Quantitative imaging of subcellular metabolism with stable isotopes and multiisotope imaging mass spectrometry. Semin Cell Dev Biol 24, 661-667.

Stroschein-Stevenson SL, Foley E, O'Farrell PH, and Johnson AD. (2009). Phagocytosis of Candida albicans by RNAitreated Drosophila S2 cells. Methods Mol Biol 470, 347-358.

Tenenhaus A, Philippe C, Guillemot V, Le Cao K-A, Grill J, and Frouin V. (2014). Variable selection for generalized canonical correlation analysis. Biostatistics 15, 569-583.

Tierney L, Linde J, Müller S, et al. (2012). An interspecies regulatory network inferred from simultaneous RNA-seq of Candida albicans invading innate immune cells. Front Microbiol 3, 85 .

Toh-e A, Ohkusu M, Li H-M, et al. (2015). Identification of genes involved in the phosphate metabolism in Cryptococcus neoformans. Fungal Genet Biol 80, 19-30.

Tom Dieck S, Kochen L, Hanus C, et al. (2015). Direct visualization of newly synthesized target proteins in situ. Nat Methods 12, 1-7.

US Food and Drug Administration. (2013). 510(k) Summary: K130010. 1-8.

Veenstra TD. (2012). Metabolomics: The final frontier. Genome Med 5, 2010-2012.

Vyas VK, Barrasa MI, and Fink GR. (2015). A Candida albicans CRISPR system permits genetic engineering of essential genes and gene families. Sci Adv 1, e1500248-e1500248.

Wächtler B, Wilson D, Haedicke K, Dalle F, and Hube B. (2011). From attachment to damage: Defined genes of Candida albicans mediate adhesion, invasion and damage during interaction with oral epithelial cells. PLoS One 6, e17046.

Wang Z, Curry E, and Montana G. (2014). Network-guided regression for detecting associations between DNA methylation and gene expression. Bioinformatics 30, 2693-2701.

Wang H, Yang H, Shivalila CS, Dawlaty MM, Cheng AW, Zhang F, and Jaenisch R. (2013). One-step generation of mice carrying mutations in multiple genes by CRISPR/casmediated genome engineering. Cell 153, 910-918.

Warnock DW. (2007). Trends in the epidemiology of invasive fungal infections. Jap J Med Mycol 48, 1-12.

Wasylnka JA, and Moore MM. (2003). Aspergillus fumigatus conidia survive and germinate in acidic organelles of A549 epithelial cells. J Cell Sci 116, 1579-1587.

Webb A. (2012). Increasing the sensitivity of magnetic resonance spectroscopy and imaging. Anal Chem 84, 9-16.

Wiese S, Reidegeld K a, Meyer HE, and Warscheid B. (2007). Protein labeling by iTRAQ: A new tool for quantitative mass spectrometry in proteome research. Proteomics 7, 340-350.

Wishart DS, Tzur D, Knox C, et al. (2007). HMDB: The Human Metabolome Database. Nucleic Acids Res 35, D521-526.

Zetsche B, Gootenberg JS, Abudayyeh OO, et al. (2015). Cpf1 is a single RNA-guided endonuclease of a Class 2 CRISPRCas system. Cell 163, 759-771.

Zhang D-S, Piazza V, Perrin BJ, et al. (2012). Multi-isotope imaging mass spectrometry reveals slow protein turnover in hair-cell stereocilia. Nature 481, 520-524.

Zhang J, Zhang L, Qiu J, and Nian H. (2015). Isobaric tags for relative and absolute quantitation (iTRAQ)-based proteomic analysis of Cryptococcus humicola response to aluminum stress. J Biosci Bioeng 120, 359-363.

Zimmermann AC, Zarei M, Eiselein S, and Dengjel J. (2010). Quantitative proteomics for the analysis of spatio-temporal protein dynamics during autophagy. Autophagy 6, 10091016.

\section{Address correspondence to: Associate Professor Scott J. Tebbutt Centre for Heart Lung Innovation University of British Columbia St. Paul's Hospital 1081 Burrard Street Vancouver \\ British Columbia V6Z 1 Y6 Canada}

E-mail: scott.tebbutt@hli.ubc.ca

$\begin{aligned} & \text { Abbreviations Used } \\ & \text { CHiP }=\text { chromatin immunoprecipitation } \text { clustered regularly interspaced short } \\ & \text { palindromic repeats } \\ & \text { CRISPR } \text { fluorescent in situ hybridization } \\ & \text { FISH } \text { fluorescent in situ sequencing } \\ & \text { FISSEQ } \text { quantitation } \\ & \text { IFI }=\text { invasive fungal infection } \text { iTRAQ }=\text { isobaric tags for relative and absolute } \\ & \text { MERFISH }=\text { multiplexed error-robust FISH } \\ & \text { MIMS }=\text { multi-isotope imaging mass spectrometry } \\ & \text { PCP-SILAC }=\text { protein correlation profiling-SILAC } \\ & \text { PUNCH-P }=\text { puromycin-assisted nascent chain } \\ & \text { proteomics } \\ & \text { RNAi }=\text { RNA interference } \\ & \text { SILAC }=\text { stable isotope labeling by amino acids } \\ & \text { in cell culture } \\ & \text { SIMS }=\text { secondary ion mass spectrometry } \\ & \text { SUnSET }=\text { surface sensing of translation }\end{aligned}$

